\title{
Correction to: Review of Robust Aerodynamic Design Optimization for Air Vehicles
}

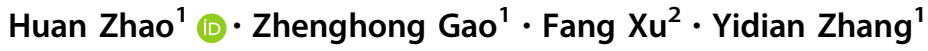

(C) CIMNE, Barcelona, Spain 2018

Correction to: Archives of Computational Methods in Engineering https://doi.org/10.1007/s11831-018-9259-2

The publisher and the authors apologize to the readership for the proofing error in Sect. 3.3.3 which has been thus recorrected in original article.
In Sect. 3.3.3, on p. 20, all the inaccurate formulae " $f(\mathbf{Z}), \psi_{i}$ " have been re-corrected with $\left\langle f(\mathbf{Z}), \psi_{i}\right\rangle$ indicating the inner product (six times).

The publisher and the authors would like to apologize for any inconvenience caused.

The original version of this article was revised.

The original article can be found online at https://doi.org/10.1007/s11831-018-9259-2.

Zhenghong Gao

zgao@nwpu.edu.cn

Huan Zhao

huanzhao_aero@163.com

1 School of Aeronautics, Northwestern Polytechnical University, Xi'an 710072, Shaanxi, People's Republic of China

2 School of Mechanical, Aerospace and Civil Engineering, University of Manchester, Manchester, UK 